\section{The effect of presenting repaired verbal or pictorial mediator prompts on chaining*}

\author{
ALLEN R. DOBBS and JEANNE-MARIE KEELING $†$ \\ University of Alberta, Edmonton, Alberta, Canada
}

One hundred Ss learned either the A-B, B-C, A-C mediation (M) or the A-D, B-C, A-C control (C) paradigm (study-test procedure) under conditions in which items on the A-C test trials were not prompted or half of the items were inappropriately prompted, with either a verbal or pictorial representation of a repaired-B term taken from the unprompted pairs. Thus, there were two prompted $M$ and $C$ groups and an unprompted $M$ and $C$ group. Inappropriate prompting led to a generalized decrement for the four prompted $M$ and $C$ groups. The decrement was equal for the verbally and pictorially prompted $\mathrm{C}$ groups. Verbal prompting led to a greater decrement for the $\mathrm{M}$ comparisons. No difference between prompted and unprompted items was obtained. The results were interpreted as being in accord with a chaining analysis of mediation but clearly contrary to the "capturing" notion previously advanced by Daehler \& Wright (1968).

The superiority of Stage III performance with the A-B, B-C, A-C mediation (M) paradigm as compared to an A-D, B-C, A-C control (C) has been explained in terms of an S's ability to utilize the prior-learned A-B and $\mathrm{B}-\mathrm{C}$ associations to mediate $\mathrm{A}-\mathrm{C}$ during Stage III learning in the $M$ paradigm. If it is assumed that mediation accounts for the facilitation obtained with the chaining paradigm, then the amount of facilitation should be a function of those variables that affect the availability of the mediating- $B$ response. Consequently, if a mediating-B item appropriate for $A_{1}-C_{1}$ (i.e., $B_{1}$ ) is presented with $A_{2}$ during the anticipation interval of the Stage III trials, it should make the appropriate mediating $\mathrm{B}_{2}$ less available and the $B_{1}$ should lead to interference due to the previously learned $B_{1}-C_{1}$ association. Popp \& Voss (1966) have obtained some support for this prediction, although it is difficult to ascertain whether any mediation occurred because a nonmediational control was not employed. Contrarily, Daehler \& Wright (1968) have reported that the presentation of a pictorial representation of a repaired mediator led to facilitation, relative to a nonmediation control, and led to no decrement as compared to a mediation condition in which no such mediator presentation was received. In this latter study, half of the items were "inappropriately prompted," with the prompts being the appropriate mediators for the remaining items. The performance on the unprompted pairs

*This research was supported by Grant APA-335 from the National Research Council of Canada. Denise Durfee and Mrs. Marva Blackmore assisted in the conduct of the ex periment.

+Now at Victoria Mental Health Centre, Victoria, British Columbia, Canada. was at the same level as the nonmediational control condition. The facilitation effect was explained in terms of a "capturing" phenomenon, i.e., the inappropriate mediator was said to be captured by the pair with which it was presented and the distinctiveness of the picture aided in the facilitation effect. Because of the rapidity with which capturing took place (it was evidenced by Trials 2-5), it was suggested that the phenomenon must be a mediational one. However, it would appear there is no evidence that laboratory acquired mediating associations have distinguished themselves by the rapidity with which they are acquired. Further, the suggestion by Daehler and Wright that the inappropriate mediator prompts did not compete with, but rather somehow "suspended," the operation of the mediational chain is clearly contrary to associative explanations of chaining.

The present investigation was undertaken to determine if the capturing phenomenon could be replicated under somewhat different conditions and when further controls were introduced. The primary procedural change was to utilize the study-test method of list presentation, in which the prompts were presented only on test trials. This method was employed because it was suspected that the capturing phenomenon may have been the result of a direct association having been acquired between the pictorial prompt and the $\mathrm{C}$ response. It is not unreasonable that such a direct association may have been acquired, since with the Daehler and Wright procedure the correct $C$ response always immediately followed the anticipation-interval presentation of the prompt. Since the study-test procedure temporally separates the test trials (on which the prompt is presented) and the study trials (on which the prompt is not presented), the utilization of this procedure severely restricted the possibility of Ss' acquiring a direct association between the mediator prompt and the C-term response.

\section{DESIGN AND SUBJECTS}

Six independent groups of Ss were employed, with three groups learning the A-B, B-C, A-C mediation (M) paradigm and three learning an $A-D$, B-C, A-C control (C) sequence. The three $M$ and $C$ sets differed only in terms of the type of B-term "mediator" prompt presented to them during the test trials of List A-C. One set was provided with no prompts $(\mathrm{N})$, another set received verbal prompts (V), and the final set was presented with pictorial prompts $(P)$. The verbal prompts were simply the printed words, whereas the pictorial prompts were line-drawing representations of the $B$ terms. For the $M$ and $C$ groups in which prompts were presented, only half of the items were prompted, with the prompts being inappropriate in that the B-term prompt appearing within the context of a prompted A-C item had previously been paired with a different $\mathrm{C}$ term during B-C learning. The A-C items which were prompted (Pr) and unprompted (UPr) were counterbalanced. Pseudoclassifications on the Pr and UPr variable were made on the A-C items for the two groups which received no prompts (MN and CN). Consequently, the overall design of the experiment was a 2 by 3 by 2 factorial, with the between-S factors being paradigm ( $\mathrm{M}$ or $\mathrm{C}$ ) and prompt type $(N, P, V)$ and the within-S factor being prompted and unprompted items.

For comparison purposes, 20 additional $S s$ learned lists conforming to an A-B, D-C, A-C unprompted control paradigm. Since the Stage III (A-C) performance of these Ss did not differ from that of Group CN (A-D, B-C, A-C), their data will not be presented.

A total of 100 University of Alberta undergraduates participated in the experiment. Participation in the research was in partial fulfillment of the introductory course requirement. Each group was equally divided in terms of males and females, and all groups contained $20 \mathrm{Ss}$, except Groups CV and CP which consisted of 10 Ss each. The males and females were assigned randomly to each group, with the restriction that each group must have $n-1$ Ss prior to the assignment of the nth $S$ to any group.

$$
\text { MATERIALS }
$$

There were eight pairs of items on each list. The A terms were Archer CVC trigrams of either $68 \%$ or $69 \%$ 
Table 1

Mean Total Correct Over the First Five A-C Trials for Prompted and Unprompted Items

\begin{tabular}{|c|c|c|c|c|c|c|c|}
\hline & & MN & MP & MV & $\mathrm{CN}$ & $\mathrm{CP}$ & $\mathrm{CV}$ \\
\hline Pr & $\begin{array}{l}\text { Mean } \\
\text { SD }\end{array}$ & $\begin{array}{r}14.30 \\
3.83\end{array}$ & $\begin{array}{r}11.25 \\
3.26\end{array}$ & $\begin{array}{l}6.95 \\
4.15\end{array}$ & $\begin{array}{r}10.40 \\
4.22\end{array}$ & $\begin{array}{l}8.70 \\
4.90\end{array}$ & $\begin{array}{l}7.70 \\
3.68\end{array}$ \\
\hline UPr & $\begin{array}{l}\text { Mean } \\
\text { SD }\end{array}$ & $\begin{array}{r}14.20 \\
4.57\end{array}$ & $\begin{array}{r}10.60 \\
3.75\end{array}$ & $\begin{array}{l}7.15 \\
4.13\end{array}$ & $\begin{array}{r}10.50 \\
3.95\end{array}$ & $\begin{array}{l}7.60 \\
4.50\end{array}$ & $\begin{array}{l}7.50 \\
4.12\end{array}$ \\
\hline
\end{tabular}

association value, and the $B$ and $C$ terms were common nouns taken from Thorndike and Lorge. The $B$ terms corresponded to drawings in the Peabody Picture Vocabulary Test, and those drawings constituted the pictorial mediators. The $\mathrm{C}$ terms were selected from the Spreen \& Schulz (1966) norms on the basis of their abstractness (mean c rating $=3.67$ ). Abstract $\mathbf{C}$ terms were employed in an attempt to reduce pictorial representations of $B$ (imagery) during B-C learning. No obvious associations existed between the items within any pair.

\section{PROCEDURE}

The materials were displayed by means of an Audiscan projector with a $17 \times 12.70 \mathrm{~cm}$ screen. The study-test method was used in the presentation of lists. In Stages I and II, the interval for both study and test items was 2 sec. In Stage III the study and test intervals were 2 and $3 \mathrm{sec}$, respectively. In an attempt to replicate the Daehler \& Wright (1968) test procedure, the $A$ term was presented alone for the first second of the test interval, whereas the prompt was presented to the right of the $A$ term for the remaining $2 \mathrm{sec}$. In conditions where there was no prompt (MN and $\mathrm{CN}$ ), the projector changed in the same manner on test trials but presented only the A term. The intertrial interval for all lists was 2 sec, and a period of approximately $3 \mathrm{~min}$ elapsed between lists. The first and second lists were continued to a criterion of two successively correct trials. If the $\mathbf{S}$ had failed to achieve the criterion in 25 trials, he was discontinued. This procedure resulted in discarding the data of eight Ss, three from the $M$ paradigm and five from the $\mathrm{C}$ paradigm. Stage III consisted of 10 trials.

Instructions for Stage III were typical for paired associate learning, except that those Ss receiving prompts were told that they would be "given a hint which may or may not help" them.

\section{RESULTS}

List I

The $\mathrm{M}$ and $\mathrm{C}$ groups learned first lists consisting of $A-B$ and $A-D$, respectively. The overall mean trials to criterion on Lists A-B for the three $M$ groups was 8.70 , and 9.03 for List A-D of the three $\mathrm{C}$ groups. The results of a 2 by 3 analysis of variance with paradigm and a pseudoclassification on prompt type revealed no statistically reliable effects $(p>.20$ for all comparisons), indicating that Lists A-B and A-D were of equal difficulty. Internal analyses indicated that the groups learning the respective lists were of equal ability.

\section{List II}

Although all groups received the same second list, differential transfer effects might be expected as a result of the differing first lists of the $M$ and $C$ groups. The mean trials to criterion ranged from 6.95 to 8.10 for the $M$ groups, with an overall mean of 7.37 , and from 4.90 to 5.90 for the $C$ groups, with an overall mean of 5.37. A 2 by 3 analysis of variance where the factors were paradigm and a pseudoclassification on prompt type showed the trend for the $M$ group means to exceed those of the $\mathbf{C}$ groups to be a reliable one, $F(1,94)=10.73$, $p<.01$. This finding indicates some negative transfer resulted from the learning of List A-B. All of the other Fs were less than 1.00 . List III

Graphs of the acquisition functions for List $\mathrm{A}-\mathrm{C}$ revealed that trials had interacted with treatment effects in that there was a ceiling effect over the last four or five trials, particularly for Groups $M N$, MP, and $C N$. Consequently, the analysis and discussion of List III will be limited to performance over the first five trials of A-C learning. Over these trials, there was only a slight tendency for the curves of the various groups to interact with trials. The prompted-unprompted variable did not interact with trials either over the first 5 or the total 10 trials. The means for the total number of correct responses over the first five trials are shown in Table 1 . As can be seen from this table, the prompted-unprompted variable had no differentiating effect. However, there is a trend for both the paradigm and prompt type variables to have been effective. These means were evaluated by means of a 2 by 3 by 2 analysis of variance with paradigm, prompt type, and a within-S comparison on prompted and unprompted items as the factors, respectively.

Statistically reliable differences were obtained for paradigm, $F(1,94)=6.78, p<.01$, and prompt type, $F(2,94)=14.04, p<.01$. The interactions between the factors prompt type and paradigm approached significance, $\quad F(2,94)=2.84$, $.10>p>.05$. The main effect of prompted-unprompted produced an $\mathrm{F}$ of less than unity, and this variable did not interact with any others. Hence, $S$ scores were collapsed over this variable in all remaining analyses.

Internal analyses with Duncan's multiple range test indicated statistically reliable mediation effects for the MN and MP conditions when compared with their respective control groups (CN and CP) at the .01 and .05 levels, respectively. Statistically, Conditions MV and CV did not differ. The trend for the various $M$ conditions to produce performance differences was confirmed statistically at the .01 level. Also, in accordance with the trends, Group CN was reliably different from both $\mathrm{CP}$ and $\mathrm{CV}$ at the .05 level, whereas these latter two conditions did not produce reliably different levels of performance.

\section{DISCUSSION}

The results of the experiment reported herein indicate two things: (1) it would appear that the phenomenon reported by Daehler \& Wright (1968) is not a mediational one, and (2) the effect of presenting an inappropriate mediator prompt for half of the A-C items results in a generalized effect, perhaps operating on the discovery or utilization phases. The discussion will focus on these two implications and each will be considered separately.

The present data are not in accord with the Daehler \& Wright (1968) "capturing" hypothesis. When the study-test method is employed, the phenomenon for which the hypothesis was advanced as an explanation disappears. This was evidenced by the decrement in mediated performance, relative to a nonprompted mediation condition, as a result of either verbal or pictorial inappropriate prompting and the ineffectiveness of the prompted-unprompted variable. Because of these findings and the support accorded the present results by Popp \& Voss (1966), there is a tendency to suggest that the phenomenon witnessed by Daehler and Wright was not, as they claimed, a variation of the chaining process. Rather, a direct association between the inappropriate prompt and the response may have been acquired in their study. If this were the case, it would seem plausible that the mediator (B) in unprompted pairs would indeed be unavailable for the chaining sequence, thus rendering performance on unprompted items to the level of the control group. That a direct $\mathrm{Br}-\mathrm{C}$ association may have been acquired in the Daehler and Wright study appears especially feasible 
because of four factors: the use of the anticipation method, long "study" intervals, concrete prompts pictorially represented, and the type of materials employed. The use of the anticipation method demanded that the C-term response was presented immediately after the inappropriate prompt. Since there was a 3 -sec study interval and a 3-sec interitem interval, the Ss effectively had $6 \mathrm{sec}$ to rehearse each Br-C pair. Further, as Wicker (1970) has noted, the use of pictorial stimuli leads to rapid acquisition. These conditions should have led to the acquisition of a $\mathrm{Br}-\mathrm{C}$ association within very few trials. Contrarily, the rapid acquisition of this association would have been unlikely in either the Popp and Voss study (because of short study intervals and the use of relatively abstract adjective prompts) or the present study (primarily because of the study-test method). In addition, whereas Popp and Voss employed words for all A, B, and C terms, Daehler and Wright employed CVCs for the $A$ terms and word $B$ and $C$ terms. Consequently, since the stimulus presented during the anticipation interval was essentially a compound stimulus composed of a relatively low-meaningful $\mathrm{CVC}$ and a pictorial representation of a high-frequency noun, on the basis of previous research (e.g., Underwood, Ham, \& Ekstrand, 1962; Weiss \& Margolius, 1954) one would expect that Ss would have had a strong tendency to stimulus select in favor of the high-meaningful element (the pictorial prompt). In addition, Solso (1968) has presented evidence suggesting that, with a compound stimulus, selection of one element of the compound stimulus is enhanced if there is a preestablished association between that stimulus component and a response term, even in the case of repairing.

In short, the Daehler \& Wright (1968) interpretation of the capturing phenomenon as being the result of a mediational (chaining) process is rejected in favor of an interpretation in terms of stimulus selection and the subsequent formation of a direct $\mathrm{Br}-\mathrm{C}$ association. In their study, the pictorial representation was most probably the functional stimulus and, because of the nature of this stimulus and the long effective study times, it is not surprising that the association was acquired "rapidly." The disappearance of the capturing phenomenon with the use of the study-test method is in full accord with this interpretation.

The finding in the present study of equivalent performance for the prompted and unprompted pairs within a list demonstrates that the presentation of an inappropriate mediator for half of the items results in a generalized decrement in performance during the mediation-test phase, as evidenced by the reliable differences obtained between the no-prompt and prompted mediation groups (MN vs MP and MV). The finding of a larger decrement for Group MV than for Group MP indicates that the greater the similarity between the repaired-B prompts and the manner in which the items were presented when the mediating associations were acquired, the greater the decrement.

It may be theoretically important to attempt to decipher the locus of the interference effect. Schulz \& Lovelace (1964) and Schulz \& Weaver (1968) have suggested that it may be fruitful to distinguish between the discovery and utilization of mediation associations. Since the prompts were never presented during the study trials in the present experiment, they should not have interfered with the "discovery" of the mediating associations nor with the acquisition of the A-C association in the case of the $\mathrm{CP}$ and $\mathrm{CV}$ control groups. Further, although the performance of the MP group was decremented relative to the no-prompt mediation group, they still showed reliable mediation when compared to their respective control (CP). It is consonant with these differences to assume that Group MP had "discovered" the mediating associations and were attempting to mediate but had difficulty retrieving the mediating associations as a result of the inappropriate prompts. That it was the retrieval process which was disrupted was also supported by an analysis of the intralist errors. Contrary to the supposition of Daehler \& Wright (1968) that the presentation of inappropriate prompts does not compete with the utilization of the previously established mediational links, the error data of the present study shows that high proportions of the intralist errors may be attributable to direct competition from B-C associations appropriate for the repaired prompts. These proportions were $.48, .69, .79$, and .44 for the prompted items of Groups MP, MV, $C P$, and $C V$, respectively. The corresponding proportions of errors for unprompted items (treated as if a prompt had been presented) were .17 , $.25, .19$, and .00 . It should be noted in this regard that both the mediation and control groups acquired B-C associations during Stage II.

Within the retrieval process, the locus of the interference is less clear. For the mediation groups, the interference may have been with the retrieval of the mediator (A-B association) or with retrieval of the B-C link of the chain. The data from the present study are most consistent with an interpretation of interference occurring at both levels.

The present investigation demonstrated that, when proper controls are employed, the prompting of a mediational sequence by the use of inappropriate prompts (i.e., repaired "mediating" $B$ terms) results in a decrement of Stage III performance under such conditions when compared with unprompted mediation conditions. It was hypothesized that repairing the mediators thus tended to create interference which rendered unavailable for retrieval appropriate responses for both prompted and unprompted items within the same list. A more pronounced decrement was detected when the prompt was presented in a manner similar to the way it was presented in earlier lists. These findings are clearly in accord with predictions from a chaining analysis of mediation.

REFERENCES
ARCHER, E. J. Re-evaluation of the
meaningfulness of all possible CVC
trigrams. Psychological Monographs, 1960, 74(10, Whole No. 497).

DAEHLER, M. W., \& WRIGHT, J. C. Visual prompting in a paired-associates mediation paradigm. Journal of Verbal Learning \& Verbal Behavior, 1968, 7 , $148-153$.

POPP, R., \& VOSS, J. F. Mediational effects as a function of Stage 3 mediator presentation. Psychonomic Science, $1966,5,133-134$.

SCHULZ, R. W., \& LOVELACE, E. A. Mediation in verbal paired-associate learning: The role of temporal factors. Psychonomic Science, 1964, 1, 95-96.

SCHULZ, R. W. \& WEAVER, G. E. The A-B, B-C, A-C mediation paradigm: The effects of variation in A-C study-and test-interval lengths and strength of $A-B$ or B-C. Journal of Experimental Psychology, 1968, 76, 303-311.

SPREEN, O., \& SCHULZ, R. W. Parameters of abstraction, meaningfulness, and pronunciability for 329 nouns. Journal of Verbal Learning \& Vexbal Behavior, $1966,5,459-468$

SOLSO, R. L. Functional stimulus selection as related to color versus verbal stimuli. Journal of Experimental Psychology $1968,78,382-387$.

UNDERWOOD, B. J, HAM, M., \& EKSTRAND, B. Cue selection in paired-associate learning. Journal of Experimental Psychology, 1962, 64, 405-409.

WEISS, W., \& MARGOLIUS, G. The effect of context stimuli on learning and retention. Journal of Experimental Psychology, 1954, 48, 318-322.

WICKER, F. W. On the locus of picture-word differences in paired-associate learning. Joumal of Verbal Learning \& Verbal Behavior. 1970, 9, 52-57. 